\title{
Study of Some Properties of Intuitionistic Fuzzy Soft Matrix
}

\author{
Mamoni Dhar \\ Department of Mathematics, Science College, Kokrajhar \\ E-mail: mamonidhar@rediffmail.com
}

\begin{abstract}
The purpose of this article is to consider the notions of intuitionistic fuzzy soft matrices(IFSM) and to study some basic results associated with such matrices. This work deals particularly with the definition and some properties of transpose of intuitionistic fuzzy soft matrices. In the process, symmetric intuitionistic fuzzy matrix is also defined and some properties are discussed. Numerical examples are provided to make the concept clear.
\end{abstract}

Index Terms-Fuzzy sets, intuitionistic fuzzy sets, intuitionistic fuzzy soft matrices, square intuitionistic fuzzy soft matrix.

\section{INTRODUCTION}

The theory of fuzzy sets introduced by Zadeh[1], showed meaningful applications in many field of studies. This idea of fuzzy sets is welcome because it handles uncertainty and vagueness which the Cantorian set cannot deal with. Fuzzy set has membership function which assigns to each element of the Universe of discourse, a number from the unit interval $[0,1]$, to indicate the degree of belongingness of the set under consideration. In the fuzzy set theory, the membership of an element to a fuzzy set is a single value between zero and one. But in reality, it may not always be true that the degree of non membership of an element in a fuzzy set is equal to one minus the membership degree because there may be some hesitation degree as well. Therefore, a generalization of fuzzy set was realized by [2], as intuitionistic fuzzy set which incorporated the degree of hesitation known as the hesitation margin (and is defined as 1 minus the sum of membership and non membership degrees respectively)

Intuitionistic fuzzy sets as generalization of fuzzy sets can be useful in situation when the description of a problem by linguistic variable, given in terms of membership function only seems to be too difficult to handle. For example, in decision making problem particularly in the case of medical diagnosis, sales analysis, new product marketing, financial services etc, there is a fair chance of a non null hesitation part in each moment of evaluation of an unknown project. To be more precise, intuitionistic fuzzy sets, let us express for example, the fact that the temperature of a patient changes and other symptoms are not quite clear.

In real life situations, most of the problems in economics, social sciences, environment etc, have various uncertainties. However, most of the existing mathematical tools for formal modeling, reasoning and computation are crisp deterministic and precise in character. There are theories namely, theory of probability, evidence, fuzzy set, intuitionistic fuzzy sets, rough sets etc for dealing with uncertainties.

Szmidt and Kacprzyk[3], showed that intuitionistic fuzzy sets are pretty useful in situations when description of a problem by linguistic variable given in terms of membership function only seems too rough. Szmidt and Kacprzyk[4], describes that due to the flexibility of IFS in handling uncertainty, they are tool for a morehuman consistent reasoning under imperfectly defined facts and imprecise knowledge . Atanassov ([5], [6]) carried out rigorous research based on the theory and applications of intuitionistic fuzzy sets.

These theories have their own difficulties as pointed out by Molodtsov[7], and as such the novel concept of soft set theory was initiated. Soft set theory has rich potential for application in solving practical problems in economics, social science, medical science etc. Maji .et.al [8], have studied the theory of fuzzy soft set. Maji. et. al [9], have extended the theory of fuzzy soft set to intuitionistic fuzzy soft sets.

Matrices play a very important role in the broad area of science and technology. However, the classical matrix theory fails to solve the problems involving uncertainties. So there was the need of expressing those uncertainties with fuzzy matrices. Yang and Ji [10], did lot of research on the fuzzy soft matrices and its application in decision making problems.

Broumi. et. al [11], have studied intuitionistic fuzzy soft sets where some some new operations were introduced while defining concentration, dialation and normalization of intuitionistic fuzzy soft sets. Chetia.et.al [12], discussed some results on intuitionistic fuzzy soft matrix theory. Verma. et. al[13], ihas introduced some new results on intuitionistic fuzzy sets by defing some operations/compositions on it. Rajarajeswari. et. al[14], has found some interesting results while dealing with intuitionistic fuzzy soft matrix theory and in applications.

Here in this article, intuitionistic fuzzy soft matrices will be considered and some properties associated with this will be studied.

The paper is organized as follows: Section I is introductory in nature. Section Ii deals with some preliminary concepts which are required in due course of this paper. Section III deals with the properties of 
transpose of intuitionistic fuzzy soft matrix. Section IV deals with symmetric intuitionistic fuzzy soft matrices and in Section V, the conclusion is provided.

\section{DENITIONS AND PRELIMINARIES}

$A$. The basic definitions of intuitionistic fuzzy set theory that are useful in subsequent sections of this article are discussed here.

\subsection{Definition: Soft set}

Suppose that $\mathrm{U}$ is an initial universe $\mathrm{P}(\mathrm{U})$ denote the power set of $\mathrm{U}, \mathrm{E}$ be the set of all parameters and $A \subseteq E$. A pair $\left(f_{A}, E\right)$ is called a soft set over $U$ and this is defined by the set of ordered pairs $\left(f_{A}, E\right)=\left\{\left(e, f_{A}(e)\right): e \in E, f_{A}(e) \in P(U)\right\}$ where $f_{A}$ is a mapping given by $f_{A}: E \rightarrow P(U)$. Clearly soft set is a mapping from parameters to $\mathrm{P}(\mathrm{U})$.

\subsection{Definition: Fuzzy soft set}

Suppose that $\mathrm{U}$ is an initial universe of discourse and $\mathrm{E}$ is the set of parameters which involves imprecise words. Let $\mathrm{P}(\mathrm{U})$ denote the power set of $\mathrm{U}$ and $A \subseteq E$. A $\operatorname{pair}\left(f_{A}, E\right)$ is called fuzzy soft set over $\mathrm{U}$ where $f_{A}$ is a mapping given by $f_{A}: E \rightarrow P(U)$.

\subsection{Definition: Intuitionistic Fuzzy soft set}

Suppose that $\mathrm{U}$ is an initial universe of discourse and $\mathrm{E}$ is the set of parameters. Let $\mathrm{P}(\mathrm{U})$ denotes the collection of all intuitionistic fuzzy subsets of $\mathrm{U}$. Let $A \subseteq E$. A pair $\left(f_{A}, E\right)$ is called an intuitionistic fuzzy soft set (IFSS) over $\mathrm{U}$ where $f_{A}$ is a mapping given by $f_{A}: E \rightarrow P(U)$.

Example: Let $\mathrm{U}$ be the set of four cities given by $U=\left\{u_{1}, u_{2}, u_{3}, u_{4}\right\}$ and $\mathrm{E}$ be the set of parameters (each parameters is an intuitionistic fuzzy word) given by $\mathrm{E}=\{$ highly, immensely, moderately, average, less $\}$ and is denoted by $E=\left\{e_{1}, e_{2}, e_{3}, e_{4}, e_{5}\right\}$, a set of parameters where

$\mathrm{e}_{1}$ stands for the parameters highly $\mathrm{e}_{2}$ stands for the parameters immensely $\mathrm{e}_{3}$ stands for the parameters moderately $\mathrm{e}_{4}$ stands for the parameters average $\mathrm{e}_{5}$ stands for the parameters less

Let $A \subseteq E$ given by $A=\left\{e_{1}, e_{2}, e_{3}, e_{5}\right\}$

Now suppose $\mathrm{f}_{\mathrm{A}}$ is the mapping defined as the polluted cities and given by

$$
\begin{gathered}
f_{A}\left(e_{1}\right)=\left\{\left(u_{1}, .2, .7\right),\left(u_{2}, .8, .1\right),\left(u_{3}, .4, .2\right),\left(u_{4}, .6, .3\right)\right\} \\
f_{A}\left(e_{2}\right)=\left\{\left(u_{2}, .9, .1\right),\left(u_{3}, .3, .6\right),\left(u_{4}, .4, .6\right)\right\} \\
f_{A}\left(e_{3}\right)=\left\{\left(u_{1}, .3, .5\right),\left(u_{2}, .4, .6\right),\left(u_{3}, .8, .1\right)\right\}
\end{gathered}
$$

$$
f_{A}\left(e_{5}\right)=\left\{\left(u_{1}, .9, .1\right),\left(u_{2}, .1, .8\right),\left(u_{3}, .5, .4\right),\left(u_{4}, .3, .5\right)\right\}
$$

Then the intuitionistic fuzzy soft set is given by

$$
\begin{aligned}
& \left(f_{A}, E\right)=\{\text { highly polluted } \\
& \text { cities } \left.=\left\{\left(u_{1}, .2, .7\right),\left(u_{2}, .8, .1\right),\left(u_{3}, .4, .2\right),\left(u_{4}, .6, .3\right)\right\}\right\}, \\
& \text { immensely polluted } \\
& \text { city }=\left\{\left(u_{2}, .9, .1\right),\left(u_{3}, .3, .6\right),\left(u_{4}, .4, .6\right)\right\}, \text { moderately } \\
& \text { polluted } \\
& \quad \text { city }=\left\{\left(u_{1}, .3, .5\right),\left(u_{2}, .4, .6\right),\left(u_{3}, .8, .1\right)\right\} \\
& \text { average polluted city }=\phi \\
& \text { less polluted } \\
& \text { city }=\left\{\left(u_{1}, .9, .1\right),\left(u_{2}, .1, .8\right),\left(u_{3}, .5, .4\right),\left(u_{4}, .3, .5\right)\right\} \phi
\end{aligned}
$$

\subsection{Definition: Intuitionistic Fuzzy Soft Matrix}

Let $\mathrm{U}$ be an initial universe and $\mathrm{E}$ be the set of parameters and $A \subseteq E$.Let $\left(f_{A}, e\right)$ be an intuitionistic fuzzy soft set over $\mathrm{U}$. Then a subset of $U \times E$ is uniquely defined by $R_{A}=\left\{(u, e): e \in A, u \in f_{A}(e)\right\}$, which is called relation form of $\left(f_{A}, e\right)$.The membership and non membership functions are defined by $\mu_{R_{A}}: U \times E \rightarrow[0,1]$ and $v_{R_{A}}: U \times E \rightarrow[0,1] \quad$ where $\mu_{R_{A}}:(u, e) \in[0,1]$ and $v_{R_{A}}:(u, e) \in[0,1]$ are membership value and non membership value of $u \in U$ and $e \in E$. Intuitionistic fuzzy soft matrix is denoted here by IFSM.

Now if the set of Universe $U=\left\{\mathrm{u}_{1}, \mathrm{u}_{2}, \mathrm{u}_{3}, \ldots \ldots, \mathrm{u}_{\mathrm{m}}\right\}$ and the set of parameters $E=\left\{e_{1}, e_{2}, e_{3}, \ldots \ldots, e_{n}\right\}$ then $R_{A}$ can be represented in tabular form in the following way:

Table 1

\begin{tabular}{|c|c|c|c|c|}
\hline & $\mathrm{e}_{1}$ & $\mathrm{e}_{2}$ & $\ldots \ldots$ & $\mathrm{e}_{\mathrm{n}}$ \\
\hline $\mathrm{u}_{1}$ & $\left(\mu_{11}, v_{11}\right)$ & $\left(\mu_{12}, v_{12}\right)$ & $\ldots \ldots$ & $\left(\mu_{1 n}, v_{1 n}\right)$ \\
\hline $\mathrm{u}_{2}$ & $\left(\mu_{21}, v_{21}\right)$ & $\left(\mu_{22}, v_{22}\right)$ & $\ldots \ldots$ & $\left(\mu_{2 n}, v_{2 n}\right)$ \\
\hline$\ldots$. & $\ldots \ldots$ & $\ldots \ldots$ & $\ldots \ldots$ & \\
\hline $\mathrm{u}_{\mathrm{m}}$ & $\left(\mu_{m 1}, v_{m 1}\right)$ & $\left(\mu_{m 2}, v_{m 2}\right)$ & $\ldots \ldots$ & $\left(\mu_{m n}, v_{m n}\right)$ \\
\hline
\end{tabular}

If $\left(\mu_{i j}, v_{i j}\right)=\left(\mu_{R_{A}}\left(u_{i}, e_{j}\right), v_{R_{A}}\left(u_{i}, e_{j}\right)\right)$, we define a matrix

$$
\left[\left(\mu_{i j}, v_{i j}\right)\right]_{m \times n}=\left[\begin{array}{cccc}
\left(\mu_{11}, v_{11}\right) & \left(\mu_{12}, v_{12}\right) & \ldots & \left(\mu_{1 n}, v_{1 n}\right) \\
\left(\mu_{21}, v_{21}\right) & \left(\mu_{22}, v_{22}\right) & \ldots & \left(\mu_{2 n}, v_{2 n}\right) \\
\ldots & \ldots & \ldots & \ldots \\
\left(\mu_{m 1}, v_{m 1}\right) & \left(\mu_{m 2}, v_{m 2}\right) & \ldots & \left(\mu_{m n}, v_{m n}\right)
\end{array}\right]
$$

which is called an $m \times n$ intuitionistic fuzzy soft matrix of the intuitionistic fuzzy soft set $\left(f_{A}, e\right)$ over $\mathrm{U}$. Therefore, we can say that the intuitionistic fuzzy soft set $\left(f_{A}, e\right)$ over $\mathrm{U}$ is uniquely characterized by the matrix $\left[\left(\mu_{i j}, v_{i j}\right)\right]_{m \times n}$. 
Example: Let $U$ be the set of four cities given by $U=\left\{u_{1}, u_{2}, u_{3}, u_{4}\right\}$ and $\mathrm{E}$ be the set of parameters (each parameters is an intuitionistic fuzzy word) given by $\mathrm{E}=\{$ highly, immensely, moderately, average $\}$ and is denoted by $E=\left\{e_{1}, e_{2}, e_{3}, e_{4}\right\}$, a set of parameters. If $A=\left\{e_{1}, e_{2}, e_{3}\right\} \subseteq E$ and $\mathrm{f}_{\mathrm{A}}$ is the mapping defined as the polluted cities and given by

$$
\begin{aligned}
& f_{A}\left(e_{1}\right)=\left\{\left(u_{1}, .4, .5\right),\left(u_{2}, .3, .5\right),\left(u_{3}, .6, .2\right),\left(u_{4}, .5, .2\right)\right\} \\
& f_{A}\left(e_{2}\right)=\left\{\left(u_{1}, .2, .5\right),\left(u_{2}, .3, .4\right),\left(u_{3}, .6, .4\right),\left(u_{4}, .3, .2\right)\right\} \\
& f_{A}\left(e_{3}\right)=\left\{\left(u_{1}, .6, .1\right),\left(u_{2}, .5, .4\right),\left(u_{3}, .3, .4\right),\left(u_{4}, .7, .2\right)\right\}
\end{aligned}
$$

Then the intuitionistic fuzzy soft set is given by

$$
\left(f_{A}, E\right)=\{\text { highly polluted }
$$$$
\text { cities } \left.=\left\{\left(u_{1}, .4, .5\right),\left(u_{2}, .3, .5\right),\left(u_{3}, .6, .2\right),\left(u_{4}, .5, .2\right)\right\}\right\} \text {, }
$$
immensely polluted

$$
\text { city }=\left\{\left(u_{1}, .2, .5\right),\left(u_{2}, .3, .4\right),\left(u_{3}, .6, .4\right),\left(u_{4}, .3, .2\right)\right\} \text {, }
$$

moderately polluted

$$
\text { city } \left.=\left\{\left(u_{1}, .6, .1\right),\left(u_{2}, .5, .4\right),\left(u_{3}, .3, .4\right),\left(u_{4}, .7, .2\right)\right\}\right\}
$$

Then the intuitionistic fuzzy set $\left(f_{A}, e\right)$ is a parameterized family $\left\{f_{A}\left(e_{1}\right), f_{A}\left(e_{2}\right), f_{A}\left(e_{3}\right)\right\}$.

Hence the intuitionistic fuzzy soft matrix can be written as

$$
\left(\mu_{i j}, \nu_{i j}\right)=\left[\begin{array}{llll}
(.4, .5) & (.2, .5) & (.6, .1) & (0,1) \\
(.3, .5) & (.3, .4) & (.5, .4) & (0,1) \\
(.6, .2) & (.6, .4) & (.3, .4) & (0,1) \\
(.5, .2) & (.3, .2) & (.7, .2) & (0,1)
\end{array}\right]
$$

\subsection{Definition: union of intuitionistic fuzzy soft matrices}

Let $A=\left[\left(\mu_{i j}^{A}, v_{i j}^{A}\right)\right], B=\left[\left(\mu_{i j}^{B}, v_{i j}^{B}\right)\right] \in \operatorname{IFSM}_{m \times n}$. Then union of $\mathrm{A}$ and $\mathrm{B}$ denoted by $A \cup B$ is defined as $A \cup B=\left[\max \left(\mu_{i j}^{A}, \mu_{i j}^{B}\right), \min \left(v_{i j}^{A}, v_{i j}^{B}\right)\right]$ for all $\mathrm{i}$ and $\mathrm{j}$.

2.6. Definition: intersection of intuitionistic fuzzy soft matrices

Let $A=\left[\left(\mu_{i j}^{A}, v_{i j}^{A}\right)\right], B=\left[\left(\mu_{i j}^{B}, v_{i j}^{B}\right)\right] \in I F S M_{m \times n}$. Then intersection of $\mathrm{A}$ and $\mathrm{B}$ denoted by $A \cap B$ is defined as $A \cap B=\left[\min \left(\mu_{i j}^{A}, \mu_{i j}^{B}\right), \max \left(v_{i j}^{A}, v_{i j}^{B}\right)\right]$ for all $\mathrm{i}$ and $\mathrm{j}$

2.7. Definition: complement of intuitionistic fuzzy soft matrix

Let $A=\left[\left(\mu_{i j}^{A}, v_{i j}^{A}\right)\right] \in \operatorname{IFSM}_{m \times n}$ then the complement of A denoted by $A^{o}$ is defined as $A^{o}=\left[\left(v_{i j}^{A}, \mu_{i j}^{A}\right)\right]$ for all $\mathrm{i}$ and $\mathrm{j}$.
2.8 Definition: max-min product of intuitionistic fuzzy soft matrices

Let $A=\left[\left(\mu_{i j}^{A}, v_{i j}^{A}\right)\right], B=\left[\left(\mu_{i j}^{B}, v_{i j}^{B}\right)\right] \in \operatorname{IFSM}_{m \times n}$. Then the max-min product of the two intuitionistic fuzzy soft matrices is denoted as $A * B$ is defined as $A * B=\left[\max -\min \left(\mu_{i j}^{A}, \mu_{i j}^{B}\right), \min -\max \left(v_{i j}^{A}, v_{i j}^{B}\right)\right]$ for all $\mathrm{i}$ and $\mathrm{j}$.

2.9. Definition: scalar multiplication of intuitionistic fuzzy soft matrix

Let $A=\left[\left(\mu_{i j}^{A}, v_{i j}^{A}\right)\right] \in I F S M_{m \times n}$ and $\mathrm{k}$ be any scalar. Then the scalar multiplication of intuitionistic fuzzy soft matrix $\mathrm{A}$ by the scalar $\mathrm{k}$ is denoted by $\mathrm{kA}$ is defined as $k A=\left[\left(k \mu_{i j}^{A}, k v_{i j}^{A}\right)\right]$, where $0 \leq k \leq 1$, for all $\mathrm{i}$ and $\mathrm{j}$.

\subsection{Definition:}

Let

$$
A=\left[\left(\mu_{i j}^{A}, v_{i j}^{A}\right)\right], B=\left[\left(\mu_{i j}^{B}, v_{i j}^{B}\right)\right] \in \operatorname{IFSM}_{m \times n}
$$

Then

$A @ B=\left\{<x, \frac{\mu_{i j}^{A}(x)+\mu_{i j}^{B}(x)}{2}, \frac{v_{i j}^{A}(x)+v_{i j}^{B}(x)}{2} \mid x \in X>\right\}$

\subsection{Definition:}

Let

$$
A=\left[\left(\mu_{i j}^{A}, v_{i j}^{A}\right)\right], B=\left[\left(\mu_{i j}^{B}, v_{i j}^{B}\right)\right] \in \operatorname{IFSM}_{m \times n}
$$

Then

$$
A \$ B=\left\{<x, \sqrt{{\mu_{i j}^{A}}^{A}(x) \mu_{i j}^{B}(x)}, \sqrt{v_{i j}^{A}(x) v_{i j}^{B}(x)} \mid x \in X>\right\}
$$

2.12. Definition: transpose of intuitionistic fuzzy soft matrix

The transpose of an intuitionistic fuzzy soft matrix of order $m \times n$ is another intuitionistic fuzzy soft matrix of order $n \times m$ obtained from a given matrix $\mathrm{A}$ by interchanging its rows and columns. Thus the intuitionistic fuzzy soft set associated with the transpose becomes a new intuitionistic fuzzy soft set over the same universe and over the same set of parameters.

Let $A=\left[\left(\mu_{i j}^{A}, v_{i j}^{A}\right)\right] \in I F S M_{m \times n}$ then the transpose of A denoted by $A^{T}$ is defined as $A^{T}=\left[\left(\mu_{j i}^{A}, v_{j i}^{A}\right)\right]$ for all $\mathrm{i}$ and $\mathrm{j}$

Example: Let us consider the above example of highly polluted cities. Here $\left(f_{A}, E\right)$ be an intuitionistic fuzzy 
soft set over the Universe $U$ and over the set of parameters E given by

$$
\begin{aligned}
& \left(f_{A}, E\right)=\{\text { highly polluted } \\
& \text { city } \left.=\left\{\left(u_{1}, .4, .5\right),\left(u_{2}, .3, .5\right),\left(u_{3}, .6, .2\right),\left(u_{4}, .5, .2\right)\right\}\right\},
\end{aligned}
$$

immensely polluted

$$
\text { city }=\left\{\left(u_{1}, .2, .5\right),\left(u_{2}, .3, .4\right),\left(u_{3}, .6, .4\right),\left(u_{4}, .3, .2\right)\right\},
$$

moderately polluted

$$
\text { city } \left.=\left\{\left(u_{1}, .6, .1\right),\left(u_{2}, .5, .4\right),\left(u_{3}, .3, .4\right),\left(u_{4}, .7, .2\right)\right\}\right\}
$$

whose associated intuitionistic fuzzy soft matrix is

$$
\left(\mu_{i j}, v_{i j}\right)=\left[\begin{array}{cccc}
(.4, .5) & (.2, .5) & (.6, .1) & (0,1) \\
(.3, .5) & (.3, .4) & (.5, .4) & (0,1) \\
(.6, .2) & (.6, .4) & (.3, .4) & (0,1) \\
(.5, .2) & (.3, .2) & (.7, .2) & (0,1)
\end{array}\right]
$$

Now the transpose of this intuitionistic fuzzy soft matrix

$$
\left(\mu_{j i}, v_{j i}\right)=\left[\begin{array}{cccc}
(.4, .5) & (.3, .5) & (.6, .2) & (.5, .2) \\
(.2, .5) & (.3, .4) & (.6, .4) & (.3, .2) \\
(.6, .1) & (.5, .4) & (.3, .4) & (.7, .2) \\
(0,1) & (0,1) & (0,1) & (0,1)
\end{array}\right]
$$

Therefore the intuitionistic fuzzy soft set associated with this transpose is

$$
\begin{aligned}
& \left(g_{A}, E\right)=\{\text { highly polluted } \\
& \text { city } \left.=\left\{\left(u_{1}, .4, .5\right),\left(u_{2}, .2, .5\right),\left(u_{3}, .6, .1\right),\left(u_{4}, 0,1\right)\right\}\right\},
\end{aligned}
$$

immensely polluted

$$
\text { city }=\left\{\left(u_{1}, .3, .5\right),\left(u_{2}, .3, .4\right),\left(u_{3}, .5, .4\right),\left(u_{4}, 0,1\right)\right\} \text {, }
$$

moderately polluted

$$
\text { city } \left.=\left\{\left(u_{1}, .6, .2\right),\left(u_{2}, .6, .4\right),\left(u_{3}, .3, .4\right),\left(u_{4}, 0,1\right)\right\}\right\}
$$

averagely polluted

city $=\left\{\left(u_{1}, .5, .2\right),\left(u_{2}, .3, .2\right),\left(u_{3}, .7, .2\right),\left(u_{4}, 0,1\right)\right\}$

The properties of transpose of intuitionistic soft fuzzy matrices will be discussed in the following section.

\section{Properties Of Transpose Of IntUitionistic FUZZY SOFT MATRIX}

A. If $\mathrm{A}, \mathrm{B}$ and $\mathrm{C}$ be three intuitionistic fuzzy soft matrices, then the following properties hold

$$
\begin{aligned}
& \text { i. }(A \cup B)^{T}=A^{T} \cup B^{T} \\
& i i .(A \cap B)^{T}=A^{T} \cap B^{T} \\
& \text { iii. }(A B)^{T}=B^{T} A^{T} \\
& \text { iv. }(k A)^{T}=k A^{T} \\
& \text { v. }((A \cup B) @ C)^{T}=(A @ C)^{T} \cup(B @ C)^{T} \\
& \text { vi. }((A \cap B) @ C)^{T}=(A @ C)^{T} \cap(B @ C)^{T}
\end{aligned}
$$

\section{Proof of Property $i$ :}

Let

$$
A=\left[\left(\mu_{i j}^{A}, v_{i j}^{A}\right)\right], B=\left[\left(\mu_{i j}^{B}, v_{i j}^{B}\right)\right], C=\left[\left(\mu_{i j}^{C}, v_{i j}^{C}\right)\right]
$$

be three intuitionistic fuzzy soft matrices. Then

$$
\begin{gathered}
A \cup B=\left[\max \left(\mu_{i j}^{A}, \mu_{i j}^{B}\right), \min \left(v_{i j}^{A}, v_{i j}^{B}\right)\right] \\
(A \cup B)^{T}=\left[\max \left(\mu_{j i}^{A}, \mu_{j i}^{B}\right), \min \left(v_{j i}^{A}, v_{j i}^{B}\right)\right]
\end{gathered}
$$

Again

$$
A^{T}=\left(\mu_{j i}^{A}, v_{j i}^{A}\right)
$$

and

$$
\begin{gathered}
B^{T}=\left(\mu_{j i}^{B}, v_{j i}^{B}\right) \\
A^{T} \cup B^{T}=\left\{\max \left(\mu_{j i}^{A}, \mu_{j i}^{B}\right), \min \left(v_{j i}^{A}, v_{j i}^{B}\right)\right.
\end{gathered}
$$

Proof of property ii:

$$
\begin{gathered}
A \cap B=\left[\min \left(\mu_{i j}^{A}, \mu_{i j}^{B}\right), \max \left(v_{i j}^{A}, v_{i j}^{B}\right)\right] \\
(A \cap B)^{T}=\left[\min \left(\mu_{i j}^{A}, \mu_{i j}^{B}\right), \max \left(v_{i j}^{A}, v_{i j}^{B}\right)\right] \\
A^{T} \cap B^{T}=\left\{\min \left(\mu_{j i}^{A}, \mu_{j i}^{B}\right), \max \left(v_{j i}^{A}, v_{j i}^{B}\right)\right.
\end{gathered}
$$

\section{Proof of Property iii:}

Let

$$
A=\left[\left(\mu_{i j}^{A}, v_{i j}^{A}\right)\right]
$$

Then

$$
\begin{gathered}
k A=\left(k \mu_{i j}^{A}, k v_{i j}^{A}\right) \\
(k A)^{T}=\left(k \mu_{j i}^{A}, k v_{j i}^{A}\right) \\
k A^{T}=\left(k \mu_{j i}^{A}, k v_{j i}^{A}\right)
\end{gathered}
$$

Hence

$$
(k A)^{T}=k A^{T}
$$

\section{Proof of Property iv:}

Again

$$
(A \cup B) @ C=\left[\left(\mu_{i j}^{A}, v_{i j}^{B}\right)\right] @\left[\left(\mu_{i j}^{C}, v_{i j}^{C}\right)\right]
$$

If 


$$
\begin{gathered}
\mu_{i j}^{A} \geq \mu_{i j}^{B} \\
v_{i j}^{A} \geq v_{i j}^{B}
\end{gathered}
$$

Then

$$
\begin{gathered}
(A \cup B) @ C=\left[\frac{\mu_{i j}^{A}+\mu_{i j}^{C}}{2}, \frac{v_{i j}^{B}+v_{i j}^{C}}{2}\right] \\
((A \cup B) @ C)^{T}=\left[\frac{\mu_{j i}^{A}+\mu_{j i}^{C}}{2}, \frac{v_{j i}^{B}+v_{j i}^{C}}{2}\right] \\
(A @ C)^{T}=\left[\frac{\mu_{j i}^{A}+\mu_{j i}^{C}}{2}, \frac{v_{j i}^{A}+v_{j i}^{C}}{2}\right] \\
(B @ C)^{T}=\left[\frac{\mu_{j i}^{B}+\mu_{j i}^{C}}{2}, \frac{v_{j i}^{B}+v_{j i}^{C}}{2}\right] \\
\mu^{A}+\mu_{i j}^{C} \geq \mu^{B}{ }_{i j}+\mu_{i j}^{C}, v_{i j}^{A}+v_{i j}^{C} \geq v_{i j}^{B}+v_{i j}^{C}, \\
(A @ C)^{T} \cup(B @ C)^{T}=\left[\frac{\mu_{j i}^{A}+\mu_{j i}^{C}}{2}, \frac{v_{j i}^{B}+v_{j i}^{C}}{2}\right]
\end{gathered}
$$

The following numerical example will illustrate the concept clearly.

B. Numerical Examples: Let us consider two fuzzy matrices $\mathrm{A}$ and $\mathrm{B}$ as follows:

$$
A=\left[\begin{array}{lll}
(.1, .2) & (.5, .4) & (.3, .6) \\
(.5, .4) & (.2, .3) & (.5, .1) \\
(.3, .6) & (.5, .1) & (.6, .2)
\end{array}\right]
$$

and

$$
B=\left[\begin{array}{lll}
(.5, .3) & (.1, .6) & (.7, .1) \\
(.8, .1) & (.4, .3) & (.5, .2) \\
(.2, .5) & (.3, .6) & (.4, .5)
\end{array}\right]
$$

Then we get

$$
\begin{gathered}
A \cup B=\left[\begin{array}{lll}
(.5, .2) & (.5, .4) & (.7, .1) \\
(.8, .1) & (.4, .3) & (.5, .1) \\
(.5, .2) & (.3, .4) & (.6, .2)
\end{array}\right] \\
A \cap B=\left[\begin{array}{lll}
(.1, .3) & (.1, .6) & (.3, .6) \\
(.4, .4) & (.2, .3) & (.5, .2) \\
(.2, .5) & (.3, .6) & (.4, .5)
\end{array}\right] \\
(A \cup B)^{T}=\left[\begin{array}{lll}
(.5, .2) & (.8, .1) & (.5, .2) \\
(.5, .4) & (.4, .3) & (.3, .4) \\
(.7, .1) & (.5, .1) & (.6, .2)
\end{array}\right]
\end{gathered}
$$




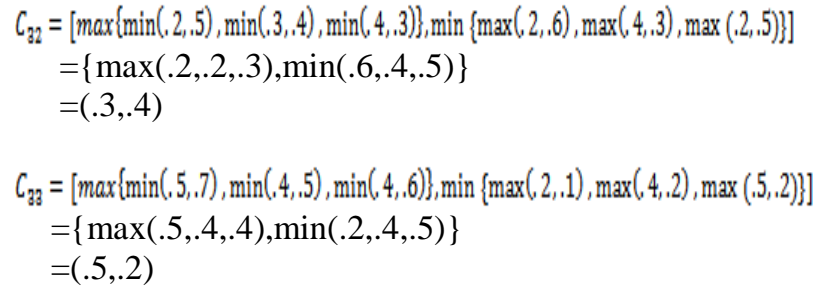

Thus we get

$$
A B=\left[\begin{array}{lll}
(.5, .3) & (.4, .4) & (.5, .2) \\
(.4, .3) & (.3, .3) & (.4, .3) \\
(.5, .3) & (.3, .4) & (.5, .2)
\end{array}\right]
$$

and

$$
(A B)^{T}=\left[\begin{array}{lll}
(.5, .3) & (.4, .3) & (.5, .3) \\
(.4, .4) & (.3, .3) & (.3,4) \\
(.5, .2) & (.4, .3) & (.5, .2)
\end{array}\right]
$$

Then let us compute $B^{T} A^{T}$ and if $B^{T} A^{T}=\left[D_{i j}\right]$, We get

$D_{11}=[\max \{\min (.5,1), \min (.8,5), \min (2,3)\}, \min \{\max (3,2), \max (1,4), \max (.5,6)\}]$ $=\{\max (.1, .5, .2), \min (.3, .4, .6)\}$ $=(.5,3)$

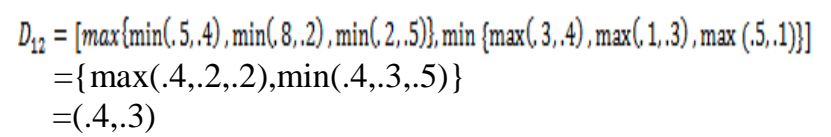

$D_{18}=[\max \{\min (.5,5), \min (8,3), \min (2,6)\}, \min \{\max (3,2,2), \max (1,4), \max (5,2,2)\}]$ $=\{\max (.5, .3, .2), \min (.3, .4, .5)\}$ $=(.5, .3)$

\footnotetext{
$D_{21}=[\max \{\min (1,1,1), \min (4,5,5), \min (3,3,3)\}, \min \{\max (6,2), \max (3,4), \max (6,6,6)\}]$ $=\{\max (.1, .4, .3), \min (.6, .4, .6)\}$ $=(.4,4)$

$D_{22}=[\max \{\min (1,4), \min (4,4), \min (3,5,5)\}, \min \{\max (6,4), \max (3,3,3), \max (6,1,1)\}]$ $=\{\max (.1, .2, .3), \min (.6, .3, .6)\}$ $=(.3,3)$

$D_{23}=[\max \{\min (1,5), \min (4,3), \min (, 3,6)\}, \min \{\max (6,2), \max (3,4), \max (.6,2)\}]$ $=\{\max (.1, .3, .3), \min (.6,4, .6)\}$ $=(.3,4)$
$D_{31}=[\max \{\min (.7,11), \min (.5,5), \min (4,3)\}, \min \{\max (1,1,2), \max (2,4), \max (.5,6)\}]$ $=\{\max (.1, .5, .3), \min (.2, .4, .6)\}$ $=(.5, .2)$

$D_{32}=[\max \{\min (7,4), \min (.5,2), \min (, 4,5)\}, \min \{\max (1,4,4), \max (2,3), \max (5,1,1)\}]$ $=\{\max (.4, .2, .4), \min (.4, .3, .5)\}$ $=(.4,3)$

$D_{38}=[\max \{\min (7,5), \min (.5,3), \min (4,6)\}, \min \{\max (1,2,2), \max (2,4), \max (5,2,2)\}]$ $=\{\max (.5, .3, .4), \min (.2, .4, .5)\}$ $=(.5,2)$
}

Hence

$$
B^{T} A^{T}=\left[\begin{array}{lll}
(.5, .3) & (.4, .3) & (.5, .3) \\
(.4, .4) & (, 3, .3) & (.3, .4) \\
(.5, .2) & (.4, .3) & (, 3, .1)
\end{array}\right]
$$

Let us chose $\mathrm{k}$ such that $k \in[0,1]$.Then we have

$$
\begin{gathered}
k A=\left[\begin{array}{ccc}
(.05, .1) & (.25, .2) & (.15, .3) \\
(.2, .2) & (.1,15) & (.25, .05) \\
(.25, .1) & (.15, .2) & (.3, .1)
\end{array}\right] \\
(k A)^{T}=\left[\begin{array}{ccc}
(.05, .1) & (.2, .2) & (.25, .1) \\
(.25, .2) & (.1,15) & (.15,2) \\
(.15, .3) & (.25, .05) & (.3, .1)
\end{array}\right]
\end{gathered}
$$

and

$$
k A^{T}=\left[\begin{array}{ccc}
(.05, .1) & (.2, .2) & (.25, .1) \\
(.25, .2) & (.1, .15) & (.15, .2) \\
(.15, .3) & (.25, .05) & (.3, .1)
\end{array}\right]
$$

Let us consider three intuitionistic fuzzy soft matrices $\mathrm{A}, \mathrm{B}$ and $\mathrm{C}$ as

$$
\begin{gathered}
A=\left[\begin{array}{lll}
(.1, .2) & (.5,4) & (.3, .6) \\
(.4, .4) & (.2, .3) & (.5, .2) \\
(.5, .2) & (.3, .4) & (.6, .2)
\end{array}\right] \\
B=\left[\begin{array}{lll}
(.5, .3) & (.1, .6) & (.7, .1) \\
(.8, .1) & (.4, .3) & (.5, .2) \\
(.2, .5) & (.3,6) & (.4, .3)
\end{array}\right] \\
C=\left[\begin{array}{lll}
(.1, .3) & (.1, .6) & (.3, .6) \\
(.4,4) & (.2,3) & (.5,2) \\
(.2, .5) & (.3, .6) & (.4, .5)
\end{array}\right]
\end{gathered}
$$

Then

$$
\begin{aligned}
A \cup B & =\left[\begin{array}{lll}
(.5, .2) & (.5, .4) & (.7, .1) \\
(.8, .1) & (.4, .3) & (.5, .1) \\
(.5, .2) & (.3, .4) & (.6, .2)
\end{array}\right] \\
(A \cup B) @ C & \\
& =\left[\begin{array}{lll}
(.3, .25) & (.3, .5) & (.5,35) \\
(.6, .25) & (.3,3) & (.5,15) \\
(.35, .35) & (.3, .5) & (.5,35)
\end{array}\right]
\end{aligned}
$$

Hence

$$
\begin{aligned}
& ((A \cup B) @ C))^{T} \\
& =\left[\begin{array}{ccc}
(.3,25) & (.6,25) & (.35,35) \\
(.3,5) & (.3,3) & (.3,5) \\
(.5,35) & (.5,15) & (.5,35)
\end{array}\right]
\end{aligned}
$$


Again

$$
A @ C=\left[\begin{array}{ccc}
(.1, .25) & (.3, .5) & (.3, .6) \\
(.4, .4) & (.2, .3) & (.5, .15) \\
(.35, .35) & (.3, .5) & (.5, .35)
\end{array}\right]
$$

and

$$
B @ C=\left[\begin{array}{ccc}
(.3, .3) & (.1, .6) & (.5, .35) \\
(.6, .25) & (.3, .3) & (.5, .2) \\
(.2, .5) & (.3, .6) & (.4, .5)
\end{array}\right]
$$

Then

$$
\begin{aligned}
& ((A @ C))^{T} \\
& =\left[\begin{array}{lll}
(.1, .3) & (.4, .45) & (.4, .35) \\
(.3, .5) & (.2, .3) & (.3, .5) \\
(.3, .6) & (.5, .2) & (.5, .35)
\end{array}\right] \\
& ((B @ C))^{T} \\
& =\left[\begin{array}{ccc}
(.3, .35) & (.6, .3) & (.25, .5) \\
(.1, .6) & (.3, .3) & (.3, .6) \\
(.5, .35) & (.5, .2) & (.4, .5)
\end{array}\right]
\end{aligned}
$$

Hence

$$
\begin{aligned}
& (A @ C)^{T} \cup((B @ C))^{T} \\
& =\left[\begin{array}{ccc}
(.3, .25) & (.6, .23) & (.35, .35) \\
(.3, .5) & (.3, .3) & (.3, .5) \\
(.5, .35) & (.5, .15) & (.3, .35)
\end{array}\right]
\end{aligned}
$$

and

$$
\begin{aligned}
& (A @ C)^{T} \cap((B @ C))^{T} \\
& =\left[\begin{array}{lll}
(.1, .35) & (.4, .45) & (.25, .5) \\
(.1, .6) & (.2, .3) & (.3, .6) \\
(.5, .6) & (.5, .2) & (.3, .5)
\end{array}\right]
\end{aligned}
$$

\section{SYMMETRIC INTUITIONISTIC FUZZY SOFT MATRIX}

A. A square intuitionistic fuzzy soft matrix A of order, will be said to be an symmetric intuitionistic fuzzy soft matrix if its transpose be equal to it, i. e $A^{T}=A$

In other words, if $A=\left[\left(\mu_{i j}^{A}, v_{i j}^{A}\right)\right] \in I F S M_{m \times n}$ and if $\mu_{i j}^{A}=\mu_{j i}^{A}$ and $v_{i j}^{A}=v_{j i}^{A}$ then the matrix $\mathrm{A}$ is said to be symmetric intuitionistic fuzzy soft matrix.

For example

Let the set of universe $U=\left\{\mathrm{u}_{1}, \mathrm{u}_{2}, \mathrm{u}_{3}, \mathrm{u}_{4}\right\}$ and the set of parameters $\mathrm{E}=\left\{\mathrm{e}_{1}, \mathrm{e}_{2}, \mathrm{e}_{3}, \mathrm{e}_{4}\right\}$. Now suppose that $A \subseteq E$ and $f_{A}: E \rightarrow P(U)$ s.t $\left(f_{A}, E\right)$ forms intuitionistic fuzzy soft set given by

$\left(\mathrm{f}_{\mathrm{A}}\left(\mathrm{e}_{1}\right)=\left\{\left(\mathrm{u}_{1}, 0.2,0.8\right),\left(\mathrm{u}_{2}, 0.3,0.7\right),\left(\mathrm{u}_{3}, 0.8,0.1\right),\left(\mathrm{u}_{4}, 0.5\right.\right.\right.$, $0.4)\}$,

$\left.f\left(e_{2}\right)=\right)=\left\{\left(u_{1}, 0.3,0.7\right),\left(u_{2}, 0.6,0.2\right),\left(u_{3}, 0.1,0.7\right),\left(u_{4}\right.\right.$, $0.7,0.2)\}$,

$f\left(e_{3}\right)=\left\{\left(u_{1}, 0.8,0.1\right),\left(u_{2}, 0.1,0.7\right),\left(u_{3}, 0.7,0.2\right),\left(u_{4}, 0.2\right.\right.$, $0.7)\}$

$f\left(e_{4}\right)=\left\{\left(u_{1}, 0.5,0.4\right),\left(u_{2}, 0.7,0.2\right),\left(u_{3}, 0.2,0.7\right),\left(u_{4}, 0.4\right.\right.$, $0.6)\}$,

The intuitionistic fuzzy soft matrix associated with these intuitionistic fuzzy soft $\operatorname{set}\left(f_{A}, E\right)$ is

$$
A=\left[\begin{array}{llll}
(0.2,0.8) & (0.3,0.7) & (0.8,0.1) & (0.5,0.4) \\
(0.3,0.7) & (0.6,0.2) & (0.1,0.7) & (0.7,0.2) \\
(0.8,0.1) & (0.1,0.7) & (0.7,0.2) & (0.2,0.7) \\
(0.5,0.4) & (0.7,0.2) & (0.2,0.7) & (0.4,0.6)
\end{array}\right]
$$

Since here $\mu_{i j}^{A}=\mu_{j i}^{A}$ and $v_{i j}^{A}=v_{j i}^{A}$

Thus A is a symmetric intuitionistic fuzzy soft matrix.

B Propositions on Symmetric intuitionistic soft fuzzy matrices

i. If $\mathrm{A}$ and $\mathrm{B}$ be two symmetric intuitionistic fuzzy matrix then $A \cup B$ is again a symmetric intuitionistic fuzzy soft matrix.

ii. If $\mathrm{A}$ and $\mathrm{B}$ be two symmetric intuitionistic fuzzy matrix then $A \cap B$ is again a symmetric intuitionistic fuzzy soft matrix.

iii. If $\mathrm{A}$ be a symmetric intuitionistic fuzzy soft matrix then $\mathrm{kA}$ is again a symmetric intuitionistic fuzzy soft matrix.

iv. If $\mathrm{A}$ and $\mathrm{B}$ be two symmetric intuitionistic fuzzy matrix then $A @ B$ is again a symmetric intuitionistic fuzzy soft matrix.

v. If $\mathrm{A}$ and $\mathrm{B}$ be two symmetric intuitionistic fuzzy matrix, then $A \$ B$ is again a symmetric intuitionistic fuzzy soft matrix.

\section{Proofs of the Propositions with examples}

\section{Proof of $i$}

If $\mathrm{A}$ and $\mathrm{B}$ be two symmetric intuitionistic fuzzy soft matrix then $A^{T}=A$ and $B^{T}=B$. Now we are to show that $A \cup B$ is again a symmetric intuitionistic fuzzy soft matrix.

We have

$$
(A \cup B)^{T}=A^{T} \cup B^{T}
$$

Since $A^{T}=A$ and $B^{T}=B$, then $A^{T} \cup B^{T}=A \cup B$ which implies that $(A \cup B)^{T}=A \cup B$ 
Thus the result follows that if $\mathrm{A}$ and $\mathrm{B}$ be two symmetric intuitionistic soft fuzzy matrices then $A \cup B$ is again a symmetric intuitionistic fuzzy soft matrix.

\section{Numerical example}

Let A and B be two symmetric intuitionistic soft fuzzy matrices such that

$$
A=\left(\begin{array}{lll}
(.1, .2) & (.5, .4) & (.3, .6) \\
(.5, .4) & (.2, .3) & (.5, .1) \\
(.3, .6) & (.5, .1) & (.6, .2)
\end{array}\right)
$$

and

$$
\begin{gathered}
B=\left(\begin{array}{lll}
(.5, .4) & (.6, .3) & (.8, .2) \\
(.6, .3) & (.7, .2) & (.4, .6) \\
(.8, .2) & (.4, .6) & (.8, .1)
\end{array}\right) \\
A \cup B=\left(\begin{array}{lll}
(.5, .2) & (.6, .3) & (.8, .2) \\
(.6, .3) & (.7, .2) & (.5, .1) \\
(.8, .2) & (.5, .1) & (.8, .1)
\end{array}\right) \\
(A \cup B)^{T}=\left(\begin{array}{lll}
(.5, .2) & (.6, .3) & (.8, .2) \\
(.6, .3) & (.7, .2) & (.5, .1) \\
(.8, .2) & (.5, .1) & (.8, .1)
\end{array}\right)
\end{gathered}
$$

From which it is clear that

$$
(A \cup B)^{T}=A \cup B
$$

\section{Proof of $i i$}

We have $(A \cap B)^{T}=A^{T} \cap B^{T}$

Proceeding in the above manner, the required result can be obtained.

\section{Proof of iii}

Let $\mathrm{A}$ be any symmetric intuitionistic fuzzy soft matrix and $\mathrm{k}$ be any scalar, and then it is to be proved that $\mathrm{kA}$ is again a symmetric intuitionistic fuzzy soft matrix. Let

$$
A=\left(\begin{array}{lll}
(.1, .2) & (.5, .4) & (.3, .6) \\
(.5, .4) & (.2, .3) & (.5, .1) \\
(.3, .6) & (.5, .1) & (.6, .2)
\end{array}\right)
$$

and $\mathrm{k}=.5$

Then

$$
k A=\left(\begin{array}{ccc}
(.05, .1) & (.25, .2) & (.15, .30) \\
(.25, .2) & (.1, .15) & (.25, .05) \\
(.15, .30) & (.25, .05) & (.30, .1)
\end{array}\right)
$$

Again

$$
(k A)^{T}=\left(\begin{array}{ccc}
(.05, .1) & (.25, .2) & (.15, .30) \\
(.25, .2) & (.1, .15) & (.25, .05) \\
(.15, .30) & (.25, .05) & (.30, .1)
\end{array}\right)
$$

This shows that

$$
(k A)^{T}=k A
$$

and hence $\mathrm{kA}$ is again a symmetric intuitionistic fuzzy soft matrix.

$$
(k A)^{T}=k A
$$

\section{Proof of iv}

If the above two intuitionistic fuzzy soft matrices are considered, then we see that

$$
\begin{gathered}
A @ B=\left(\begin{array}{ccc}
(.3, .3) & (.55, .35) & (.55, .4) \\
(.55, .35) & (.45, .25) & (.45, .35) \\
(.55, .4) & (.45, .35) & (.65, .2))
\end{array}\right) \\
(A @ B)^{T}=\left(\begin{array}{ccc}
(.3, .3) & (.55, .35) & (.55, .4) \\
(.55, .35) & (.45, .25) & (.45, .35) \\
(.55, .4) & (.45, .35) & (.65, .2))
\end{array}\right)
\end{gathered}
$$

and the above result shows that intuitionistic fuzzy soft matrix $A @ B$ is again a symmetric intuitionistic fuzzy soft matrix.

\section{Proof of $v$}

If the above two intuitionistic fuzzy soft matrices are considered, then we see that

$$
A \$ B=\left(\begin{array}{lll}
(\sqrt{.05}, \sqrt{.08}) & (\sqrt{.30}, \sqrt{.12}) & (\sqrt{.24}, \sqrt{.12}) \\
(\sqrt{.30}, \sqrt{.12}) & (\sqrt{.14}, \sqrt{.06}) & (\sqrt{.20}, \sqrt{.06}) \\
(\sqrt{.24}, \sqrt{.12}) & (\sqrt{.20}, \sqrt{.06}) & (\sqrt{.40}, \sqrt{.03})
\end{array}\right)
$$

Here

$$
(A \$ B)^{T}=\left(\begin{array}{lll}
(\sqrt{.05}, \sqrt{.08}) & (\sqrt{.30}, \sqrt{.12}) & (\sqrt{.24}, \sqrt{.12}) \\
(\sqrt{.30}, \sqrt{.12}) & (\sqrt{.14}, \sqrt{.06}) & (\sqrt{.20}, \sqrt{.06}) \\
(\sqrt{.24}, \sqrt{.12}) & (\sqrt{.20}, \sqrt{.06}) & (\sqrt{.40}, \sqrt{.03})
\end{array}\right)
$$

This implies $(A \$ B)^{T}=A \$ B$ and hence $A \$ B$ is again a symmetric intuitionistic fuzzy soft matrix.

\section{CONCLUSION}

Here the main intention was to study the transpose of intuitionistic fuzzy matrices and symmetric intuitionistic fuzzy soft matrices. Some of the properties of transpose 
of intuitionistic fuzzy soft matrices have been discussed and verified with numerical examples. Further, symmetric intuitionistic soft fuzzy matrices are defined and some properties associated with symmetric intuitionistic fuzzy matrices are discussed with numerical examples. It is seen that some properties of transpose of intuitionistic fuzzy soft matrices and also those of symmetric intuitionistic fuzzy matrices coincides with those of classical matrices and fuzzy matrices. In future, the main concentration will be in the direction of considering all the other properties of classical matrices in terms of intuitionistic fuzzy soft matrices.

\section{ACKNOWLEDGEMENT}

The author is grateful to all the referees for their valuable suggestions in the preparation of the article.

\section{REFERENCES}

[1] Zadeh, L.A., Fuzzy Sets, Information and Control, 1965, 8 , 338-353.

[2] Atanassov, K.T., "Intuitionistic Fuzzy Sets", Fuzzy Sets and Systems, 1986, 20(1), 87-96.

[3] Szmidt, E., Kacprzyk, J., "Intuitionistic fuzzy sets in some medical applications", Note on IFS, 2001, 7 (4), 58-64.

[4] Szmidt, E., Kacprzyk, J., "Medical diagnostic reasoning using a similarity measure for intuitionistic fuzzy sets", Note on IFS, 2004, 10 (4), 61-69.

[5] Atanassov, K.T., "Intuitionistic fuzzy sets: theory and application", Springer, 1999.

[6] Atanassov, K.T., "On Intuitionistic fuzzy sets", Springer 2012.

[7] Molodtsov, D.A., "Soft Set Theory First Results" Computers and Mathematics with Applications, 1999, 37, $19-31$.
[8] Maji, P.K., Biswas, R., and Roy, A.R., "Soft Set Theory", Computer and Mathematics with Applications, 2003, 45, 555-562.

[9] Maji, P.K., Biswas, R., and Roy, A.R, "Intuitionistic Fuzzy Soft Sets", Journal of Fuzzy Mathematics, 2001, 9, 677-692.

[10] Yong Yang and Chenli, Ji, "Fuzzy Soft Matrices and their Applications", Part 1, LNAI, 2011, 618-627, 7002.

[11] Broumi, S., Smarandache, F., Dhar, M., and Majumbar, P., "New Results of Intuitionistic Fuzzy Soft Sets", International Journal of Enjgineering and Electronic Business, 2014, 2, 47-52.

[12] Chetia, B., Das, P. K., "On Fuzzy Soft Matrix Theory", Journal of Assam Academy of Mathematics, 2010, Vol.2, 71-83.

[13] Verma, R., Dev Sharma, B., "Some new Results on Intuitionistic Fuzzy Sets", Proceeding of the Jangjeon Mathematical Society, 2013, 16(1), 101-114

[14] Rajarajeswari, P., Dhanalakshmi, P., Intuitionistic fuzzy soft matrix theory and its application in decision making, International Journal of Engineering Research \& Technology, 2013, 2 (4).

\section{Authors' Profiles}

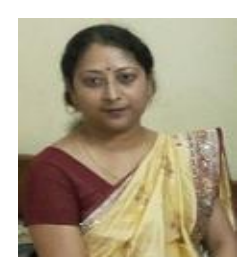

Mamoni Dhar is an Assiatant Professor in the Department of Mathematics, Science College, Kokrajhar. She obtained her Ph.D from Gauhati University, Assam, India. Her research interest is in the theory of fuzzy sets.

How to cite this paper: Mamoni Dhar,"Study of Some Properties of Intuitionistic Fuzzy Soft Matrix", International Journal of Information Engineering and Electronic Business(IJIEEB), Vol.8, No.2, pp.37-45, 2016. DOI: 10.5815/ijieeb.2016.02.05 